\title{
Electrochemical detection of adrenaline and hydrogen peroxide on carbon nanotubes
}

\section{Gaurang Khot}

Department of Neurophysiology, Donders Institute for Brain, Cognition and Behavior, Radboud University, Nijmegen, the Netherlands; Faculty of Technology and Bionics, Rhine-Waal University of Applied Sciences, Kleve, Germany

\section{Mohsen Kaboli}

Robotics, Tactile and Artificial Intelligence, Bayerische Motoren Werke AG (BMW), Munich, Germany

\section{Tansu Celikel}

Department of Neurophysiology, Donders Institute for Brain, Cognition and Behavior, Radboud University, Nijmegen, the Netherlands

Neil Shirtcliffe

Faculty of Technology and Bionics, Rhine-Waal University of Applied Sciences, Kleve, Germany (corresponding author:

neil.shirtcliffe@hochschule-rhein-waal.de)

Adrenaline and hydrogen peroxide $\left(\mathrm{H}_{2} \mathrm{O}_{2}\right)$ have neuromodulatory functions in the brain, and peroxide is also formed during reaction of adrenaline. Considerable interest exists in developing electrochemical sensors that can detect their levels in vivo due to their important biochemical roles. Challenges associated with the electrochemical detection of hydrogen peroxide and adrenaline are that the oxidation of these molecules usually requires highly oxidising potentials (beyond $1.4 \mathrm{~V}$ against silver $(\mathrm{Ag})$ /silver chloride $(\mathrm{AgCl})$ ) where electrode damage and biofouling are likely and the signals of adrenaline, hydrogen peroxide and adenosine overlap on most electrode materials. To address these issues, the authors fabricated pyrolysed carbon (C) electrodes coated with oxidised carbon nanotubes. Using these electrodes for fast-scan cyclic voltammetric measurements showed that the electrode offers reduced overpotentials compared with graphite and improved resistance to biofouling. Adrenaline oxidises on this electrode at $0.75( \pm 0.1) \mathrm{V}$ and reduces back at $-0.2( \pm 0.1) \mathrm{V}$, while hydrogen peroxide oxidation is detected at $0.85( \pm 0.1) \mathrm{V}$ on this electrode. The electrodes are highly sensitive with a sensitivity of $16 \mathrm{nA} / \mu \mathrm{M}$ for adrenaline and $11 \mathrm{nA} / \mu \mathrm{M}$ for hydrogen peroxide on an $80 \mu^{2}$ electrode. They are also suitable for distinguishing between adrenaline, hydrogen peroxide and adenosine. Thus, these probes can be used for multimodal detection of analytes.

Keywords: adrenaline/biochemical/biosensor/CNT/FSCV/hydrogen peroxide/surfaces

\section{Introduction}

Fast-scan cyclic voltammetry (FSCV), where the voltage sweep is extremely fast, has received considerable attention for the detection of neurotransmitters and neuromodulators, as it increases sensitivity and measurement speed. ${ }^{1,2}$ The ability of FSCV to detect nanomolar concentrations of a target analyte using chemically modified electrodes ${ }^{3}$ is of particular interest to neuroscientists, as it allows the detection of physiologically relevant concentrations of various bioactive substances in the living brain. ${ }^{2}$ Challenges associated with the development of chemically modified electrodes as brain implants are the degradation of chemical probes over time, loss of sensitivity, overlapping signals from analytes, biofouling and immune responses at the site of implantation. ${ }^{2}$ These issues have limited the application of carbon (C) fibre electrodes, which are considered to be the gold standard material for in vivo and in vitro preclinical applications because other conventional materials have even less favourable properties. ${ }^{2}$ Moreover, carbon fibre electrodes commonly cannot resolve dopamine and serotonin, ${ }^{4}$ hydrogen peroxide $\left(\mathrm{H}_{2} \mathrm{O}_{2}\right)$ and adenosine from one another, ${ }^{2,5,6}$ which limits their application in biochemically complex environments such as the cerebrospinal fluid.

Considerable interest exists in using carbon nanotubes (CNTs) as an alternative electron carrier. Thanks to their small size, faster electron-transfer kinetics, reduced overpotentials, biostability and resistance to biofouling, CNTs have become an attractive material for the electrochemical detection of neurotransmitters and neuromodulators. ${ }^{2}$ Due to their hydrophobic nature and $\pi$-bonding, they form aggregates when dispersed in most solvents. ${ }^{7}$ To address these issues, the functionalisation of CNTs can be performed with oxidising acids, which opens the ends of the CNTs and grafts oxygen $(\mathrm{O})$ atoms onto the exposed edge planes, making them polar enough to disperse in alcohols and even water. ${ }^{7}$ This functionalisation also allows CNTs to act as a local proton donor, thereby reducing overpotential for redox reactions in which proton transfer is required. ${ }^{7}$ CNT-based surfaces have been used in electrochemistry for electrochemical detection of neurotransmitters and neuromodulators. ${ }^{8-12}$

Neuromodulators are an important class of compounds that manipulate neuronal activity - for example, by altering the firing pattern of neurons. ${ }^{1}$ Adrenaline is a monoamine neurotransmitter that serves a dual purpose, both as a hormone and as a neurotransmitter. ${ }^{13}$ In the brain, adrenaline is responsible for various cognitive functions, including alertness and flight-or-fight response. ${ }^{14}$ Dysregulation of adrenaline is known to cause depression and anxiety. ${ }^{14}$

Hydrogen peroxide, superoxide $\left(\cdot \mathrm{O}_{2}\right)$ and hydroxyl radicals $(\cdot \mathrm{OH})$ are generated as the end product of energy metabolism and are usually considered to be waste/toxic materials to cells. Recent 
evidence suggests that peroxide molecules also play a significant role in cellular signalling similar to neurotransmission. ${ }^{15-17}$ The dual nature of hydrogen peroxide as a neuromodulator and in energy generation makes being able measure it attractive.

Electrochemical detection of adrenaline on carbon electrodes is reported at a highly anodic potential above $+1.3 \mathrm{~V} .^{13,18}$ The use of such highly oxidising potential causes degradation of electrodes and generation of reactive oxygen species at the surface of the electrode, thereby reducing the sensitivity of electrodes. ${ }^{13}$ These challenges have limited the ability to measure adrenaline in the brain. Another issue with adrenaline detection in the brain is interference from analytes such as hydrogen peroxide and adenosine, which have overlapping signals. ${ }^{1,19,20}$ Thus, the occurrence of identical signals for multiple analytes requires electrode materials on which the signals from these analytes can be resolved. ${ }^{21}$ Studies have shown that CNTs have faster electrontransfer kinetics than graphite, $^{12}$ are resistant to biofouling particularly when oxidised ${ }^{22}$ and are able to discriminate between these analytes, as the overpotentials for monoamines are considerably different from what they are on graphite. ${ }^{4}$ Biofouling is an issue with adrenaline, as it has a tendency to polymerise when oxidised to form melanin, an insoluble polymer that precipitates on the surface. ${ }^{23,24}$ The authors here therefore exploited the favorable properties of oxidised CNTs, by coating them onto pyrolytic carbon electrodes to investigate the electrochemical dynamics of adrenaline and hydrogen peroxide. The authors here also applied FSCV, as this has proved capable of measuring the concentrations of similar compounds at speeds relevant to in vivo application. ${ }^{25}$

A number of previous studies ${ }^{26-31}$ have investigated similar materials for the electrochemical detection of adrenaline and similar compounds. In most cases, these have been aiming at in vitro measurements with the potential application being urine. This allows the use of large-area electrodes, soft electrodes and toxic components. Here the authors have focused on developing an ultramicroelectrode that is stiff enough to insert into tissue and stay away from potentially unsafe materials. Nasirizadeh et al. ${ }^{26}$ used a similar approach to that used here, but with a much larger electrode and a different polymer matrix, as where they wished to measure the concentration of ascorbate, the authors here wished to exclude it. Unlike previous groups, the authors here are specifically working towards implantable sub-micrometre electrodes for measuring neurotransmitters in the presence of ascorbate fast enough to detect single releases. This publication represents a step in that direction.

\section{Materials and methods}

Hydrogen peroxide, hydrochloric $(\mathrm{HCl})$ and sulfuric acids $\left(\mathrm{H}_{2} \mathrm{SO}_{4}\right)$, adrenaline, 4-(2-hydroxyethyl)-1-piperazineethanesulfonic acid, sodium chloride $(\mathrm{NaCl})$, potassium chloride $(\mathrm{KCl})$, sodium bicarbonate $\left(\mathrm{NaHCO}_{3}\right)$, magnesium chloride $\left(\mathrm{MgCl}_{2}\right)$, monosodium phosphate $\left(\mathrm{NaH}_{2} \mathrm{PO}_{4}\right)$ and Nafion $1100 \mathrm{~W}$ (5\% in alcohols) were sourced from Sigma-Aldrich, Germany. Quartz capillaries (outer diameter (o.d.) $1 \mathrm{~mm}$, inner diameter (i.d.) $0.5 \mathrm{~mm}$, length $7.5 \mathrm{~cm}$ ) were purchased from Sutter Instrument. The water used in the experiments was deionised in-house using a Millipore column and had a resistivity of $18 \mathrm{M} \Omega$

\subsection{Fabrication of microelectrodes}

Quartz capillaries (o.d. $1.0 \mathrm{~mm}$ and i.d. $0.5 \mathrm{~mm}$ ) were pulled using a Sutter P-2000 puller (Sutter Instrument). The pulling parameters were as follows: heating temperature $750^{\circ} \mathrm{C}$, velocity 50 and DEL 127 (instrument parameters), which gave a tip diameter of $0.8-1.5 \mu \mathrm{m}$. The tip was broken shorter to reach a final diameter of $25-30 \mu \mathrm{m}$. Other micropipette pullers will have their own parameters, but the main difference from normal micropipettes is the melting point of quartz.

\subsection{Pyrolysis}

Pyrolysed carbon microelectrodes were fabricated by performing pyrolysis of propane in a nitrogen environment. Standard cooking propane was fed into the micropipette at the large end at atmospheric pressure plus 1 bar. The other end was bathed in a flow of nitrogen from a cylinder flowing out of a wide quartz tube at $50 \mathrm{ml} / \mathrm{min}$ and heated with a small butane/air torch. Quartz capillaries were heated for $1 \mathrm{~min}$ at the narrower end for carbonisation. Once the desired duration had been reached, the heat was moved towards the broader end. Upon completion of pyrolysis, the electrodes were allowed to cool under nitrogen flow. The micropipette was held at a dull orange heat, which is why quartz was required for this step.

\subsection{Dispersion of CNTs into the Nafion solution}

CNTs were functionalised by dispersing them in concentrated hydrochloric acid and concentrated sulfuric acid at a ratio of $3: 1$ $(\mathrm{v} / \mathrm{v})$. The solution was stirred with a magnetic stirrer for $48 \mathrm{~h}$. This served to open the ends of the tubes, remove any metal catalyst and graft carboxylic acid groups to the edges. Washing was carried out by decanting off the acid and repeated washing and decanting with distilled water until $\mathrm{pH} 7.0$ was reached. After washing, the CNTs were allowed to air-dry for 24-36 h. A similar method has been shown to generate carboxylate and alcohol groups at CNTs by previous researchers, ${ }^{32}$ although the authors were unable to detect these groups directly. The treated CNTs wetted with water and were dispersible in ethanol; untreated CNTs were not, due to their low surface energy.

The oxidised CNTs were suspended in a solution of Nafion ${ }^{\circledR}$ diluted with ethanol and sonicated for $30 \mathrm{~min}$ prior to use. The suspension was stable for 6-8 weeks. To obtain a thin coating of oxidised CNTs onto the electrode, the pyrolytic carbon electrodes were dip-coated in this solution for $5 \mathrm{~s}$, shaken off and allowed to dry. On larger glassy carbon electrodes for reference, this treatment changed them optically from shiny black to dull black and the water contact angle from $70^{\circ}$ to totally spreading. The coating was just about visible in field emission gun scanning electron microscopy images. However, owing to the very small size of the CNTs and their sharp tips, the authors were unable to image them successfully. The process allowed the 
Electrochemical detection of adrenaline and hydrogen peroxide on carbon nanotubes

Khot, Kaboli, Celikel and Shirtcliffe deposition of a sufficient amount of CNTs to modify the surface properties of the electrode. Successful coating could be verified by a change in electrochemical signal in a phosphate buffer solution, as the tips of the micropipettes are too small for the change to be seen easily by eye.

\subsection{Electrochemical set-up}

Electrochemical detection of adrenaline and hydrogen peroxide was carried in an electrophysiological set up that had ports for delivery and outflow of solution. ${ }^{31,33-36}$ To mimic the rapid change in concentrations observable in the brain, a flow cell was set up that delivered the target analyte as a $5 \mathrm{~s}$ bolus, followed by a pause of $10 \mathrm{~s}$. A constant flow of buffer was maintained at $2 \mathrm{ml} / \mathrm{min}$ serving as a background. Electrochemical detection of analytes was carried out using the 'dopamine waveform', ${ }^{21}$ wherein the electrode was cycled from -0.4 to $1.3 \mathrm{~V}$ and cycled back to $-0.4 \mathrm{~V}$ at $400 \mathrm{~V} / \mathrm{s}$ in the presence of a silver $(\mathrm{Ag})$ wire, coated in silver chloride $(\mathrm{AgCl})$ in a potassium chloride solution $(3.5 \mathrm{M})$ serving as a reference electrode (silver/ silver chloride). Data were collected and stored offline for analysis.

\section{Results and discussion}

\subsection{Electrochemical detection of adrenaline on pyrolytic-CNT electrodes}

The electrochemical behaviour of adrenaline on CNT-coated electrodes was studied by introducing $1 \mu \mathrm{M}$ of adrenaline to an electrode being voltage scanned. The electrode was exposed to

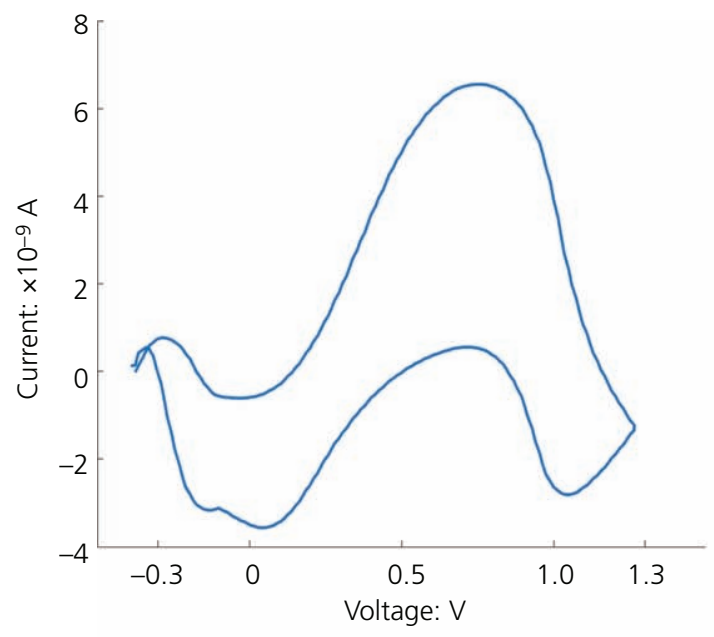

(a)

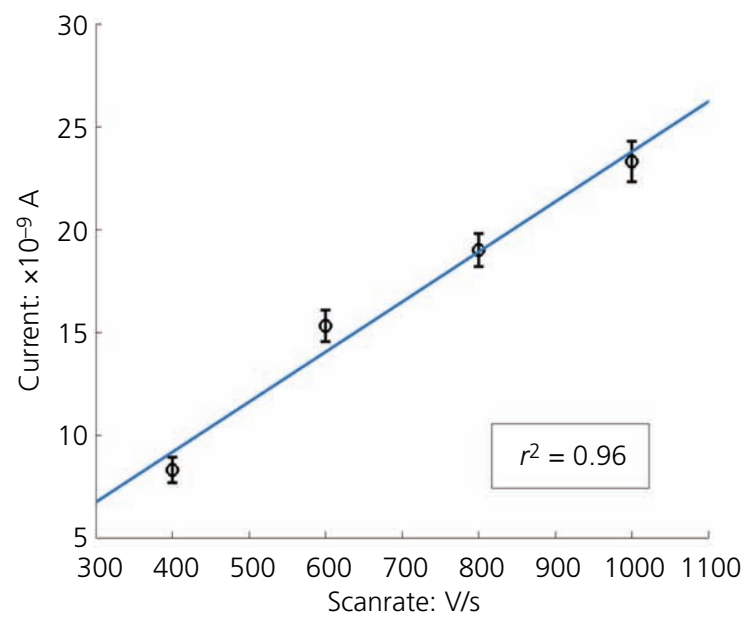

(c)

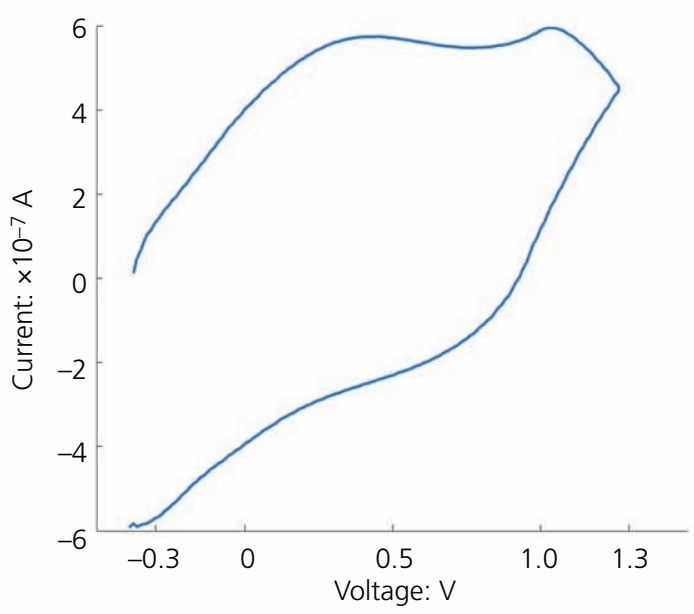

(b)

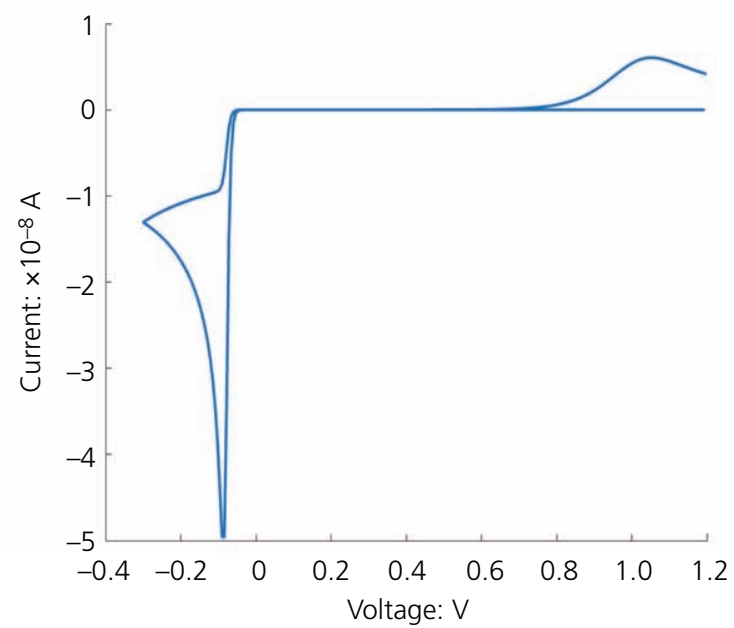

(d)

Figure 1. Oxidation of $1 \mu \mathrm{M}$ adrenaline from -0.4 to $1.3 \mathrm{~V}$ at $600 \mathrm{~V} / \mathrm{s}$ and $10 \mathrm{~Hz}$; adrenaline is oxidised at $0.75( \pm 0.1) \mathrm{V}$ and reduced at -0.2 $( \pm 0.1) \mathrm{V}$. (b) Background current. The oxidation peaks at 0.4 and $1.1 \mathrm{~V}$ and the reduction peak at $0.9 \mathrm{~V}$ are likely to be due to surface groups formed during oxidation of carbon. ${ }^{39}$ (c) The peak current is directly proportional to the scan rate with an $r^{2}=0.96$ fit, showing that the current is dominated by adsorbed species. The electrode has a sensitivity of $16 \mathrm{nA} / \mu \mathrm{M}$ on about $80 \mu \mathrm{m}^{2}$. (d) Theoretical prediction for adrenaline, using a diffusion constant of $6 \times 10^{-6} \mathrm{~m}^{2} / \mathrm{s}$. The rate of electron transfer was kept at $1 \times 10^{-6} \mathrm{~m}^{2} / \mathrm{s}$; other parameters are standard 
ten consecutive cycles of adrenaline (ten bolus additions) while cycling the potential, initially at $400 \mathrm{~V} / \mathrm{s}$. Multiple oxidation peaks and one reduction peak were observed in the cyclic voltammograms at this scan rate. One oxidation peak $(0.9 \mathrm{~V})$ was associated with oxidation of adrenaline, and a second peak was seen at $1.1 \mathrm{~V}$. Monoamines, such as dopamine and serotonin, are known to polymerise onto surfaces, usually from the partially oxidised intermediate. This generates a non-conductive surface layer, which on sensors leads to loss in sensitivity, although adrenaline is not as bad. ${ }^{37,38}$ Hence, the authors chose to increase the scan rate to $600 \mathrm{~V} / \mathrm{s}$ to limit this side reaction. Backgroundsubtracted voltammograms show an oxidation peak for adrenaline at $0.75( \pm 0.1) \mathrm{V}$, and the product is probably reduced back at -0.2 $( \pm 0.1) \mathrm{V}$, although this peak is difficult to resolve from the background (Figure 1(a)). This is a difference voltammogram with the baseline (Figure 1(b)) measured in plain buffer. A broad oxidation peak is seen, suggesting that the oxidation of adenosine on pyrolytic-CNT electrodes is a multistep oxidation process occurring on the surface of the electrode.

The authors further investigated the behaviour of adrenaline on the electrode surface, by varying the scan speed and keeping the concentration of adrenaline constant. As the scan rate was varied, the peak current increased linearly, suggesting that adrenaline binds to the surface of the electrodes (Figure 1(c)). This type of pre-adsorption increases the sensitivity of detection by increasing the amount of analyte available to the electrode for a short time, thereby allowing the detection of the nanomolar concentration of the analyte of interest. ${ }^{21,40}$ To investigate if this waveform could be used for longer-term measurement, the electrode was exposed to ten consecutive cycles of adrenaline for $10 \mathrm{~min}$. No changes in the voltammogram over this time were observed, suggesting that the CNT surface combined with the increased scan rate limits the polymerisation of adrenaline onto CNT electrodes, thus providing long-term stability. To understand the oxidation of adrenaline on CNT electrodes better, the authors modelled the rate of electron transfer, diffusion coefficient and chemical rate constant. Mathematical modelling and previous work on glassy carbon electrodes $^{40}$ suggest that adrenaline is oxidised at $1.1 \mathrm{~V}$ and reduced at $-0.15 \mathrm{~V}$ against silver/silver chloride (Figure 1(d)). Experimentally, oxidation is measured at $0.75 \mathrm{~V}$. The difference between theoretical and experimental data can be accounted for by heteroatoms that are expected to be present on the surface of the electrode shifting the voltage. This has been suggested before by other researchers, ${ }^{27,28,30}$ even the effect of abrasion can cause a shift in voltage.$^{41-43}$ The magnitude of the current is difficult to model due to the likelihood of adsorption, the high specific surface area of CNTs and the way that the quartz capillaries break increasing the surface area. Example images of tips are shown in Figures 2(c) and 2(d), revealing that the break is typically at an angle to the short axis of the capillary, resulting in a higher end area.

Comparing these results with a few other recent publications (Table 1) detecting adrenaline, the specific sensitivity per unit area is comparable with them, several of which performed well in comparison with other results. As the limit of detection depends on the electronics as well as the chemistry and size of the electrode, it is difficult to compare these values fairly. The requirement for sensitivity is particularly acute in electrodes for implantation or scanning over a surface, as their surface area cannot be increased without impacting their function in other ways.

\subsection{Mechanism of adrenaline oxidation}

Electrochemical investigations allow sub-second detection of analytes of interest. ${ }^{44}$ The method also gives some insight into the electron transport process. To visualise the excitation of molecules under investigation, the redox current was plotted against the number of cycles and current in the form of two-dimensional hotspots (Figure 2(a)). This map allows tracking the electrontransfer kinetics of adrenaline. The plot shows an oxidation signature at $0.75 \mathrm{~V}$ and a second oxidation signature at $0.9 \mathrm{~V}$. The reduction signal at $1.1 \mathrm{~V}$ (also in Figure 1(a)) has not been properly identified and is most likely the reduction of a side product of the reaction such as adrenochrome or an intermediate in the formation of peroxide and melanin. This observation is consistent with the authors' previous recording of dopamine and serotonin on CNT and pyrolytic carbon surfaces (not shown), which shows similar behaviour on oxidative reaction. A reduction signature is seen at $-0.2 \mathrm{~V}$. From this, it is clear that adrenaline oxidation on CNT-pyrolytic electrodes is not a single step and consists of multiple intermediates.

To explain the electrochemical behaviour of adrenaline on CNT electrodes, the authors applied the 'ECE' model presented by Lin et $a l^{45}$ and Bacil et $a l^{46}$ A likely mechanism of adrenaline oxidation is the loss of an electron followed by rapid deprotonation. An intermediate radical is formed, which loses a further electron to form the quinone product. ${ }^{47}$ Figure 2(b) shows a simulated voltammogram with these parameters compared with the measured data. While the peak current for experimental data is higher than those of the simulation, this is probably due to the shape of the break of the quartz capillaries increasing the effective area (Figures 2(c) and 2(d)). Both peaks are far broader than the simulated ones, and the reduction peak shows several maxima, suggesting a multistage oxidation and reduction process.

\subsection{Selectivity and specificity}

During the normal polymerisation of adrenaline (and other monoamines) to melanin, peroxides are formed. ${ }^{48}$ For this reason, it is useful to be able to measure peroxide and adrenaline simultaneously.

To evaluate if the pyrolytic-CNT electrode is capable of distinguishing analytes, the electrode was exposed to hydrogen peroxide and adenosine. Hydrogen peroxide is known to be oxidised at $1.4 \mathrm{~V}$ on glassy carbon electrodes. ${ }^{5,6}$ Background-subtracted voltammetry shows the oxidation of hydrogen peroxide on the surface of the CNT electrode (Figure 3(a)) where hydrogen peroxide is oxidised at $0.85 \mathrm{~V}$ and has no reduction peak. To understand the electrochemical kinetics 


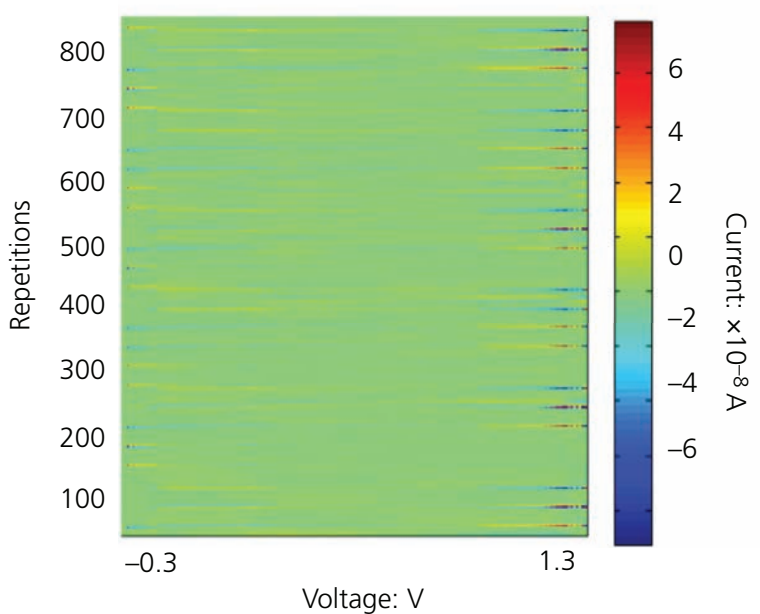

(a)

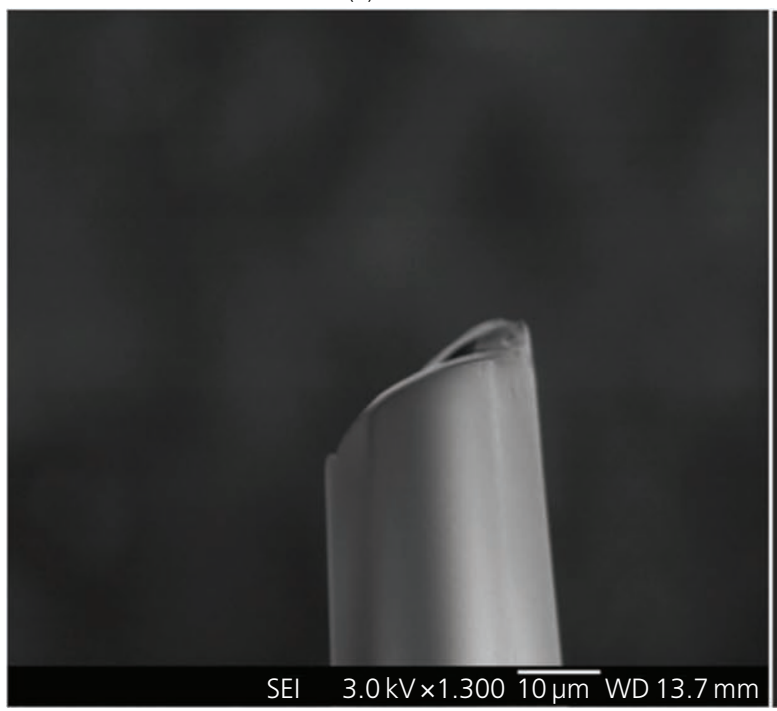

(c)

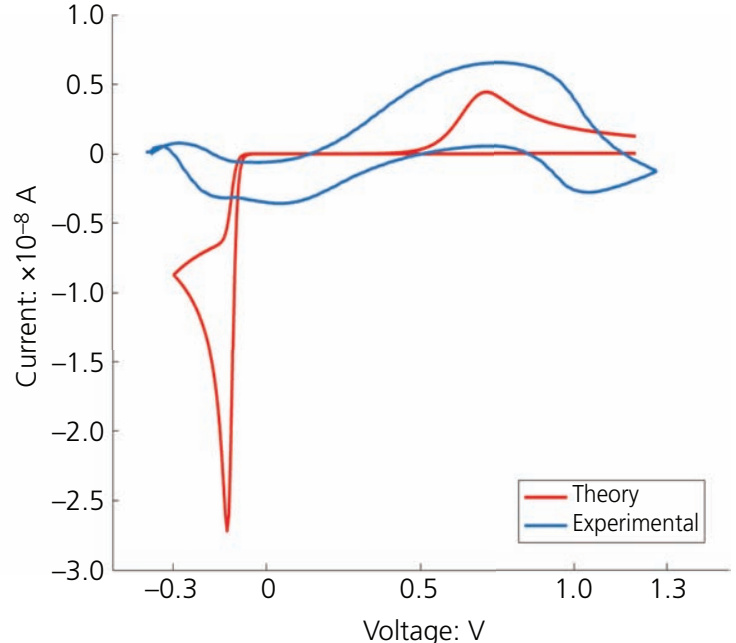

(b)

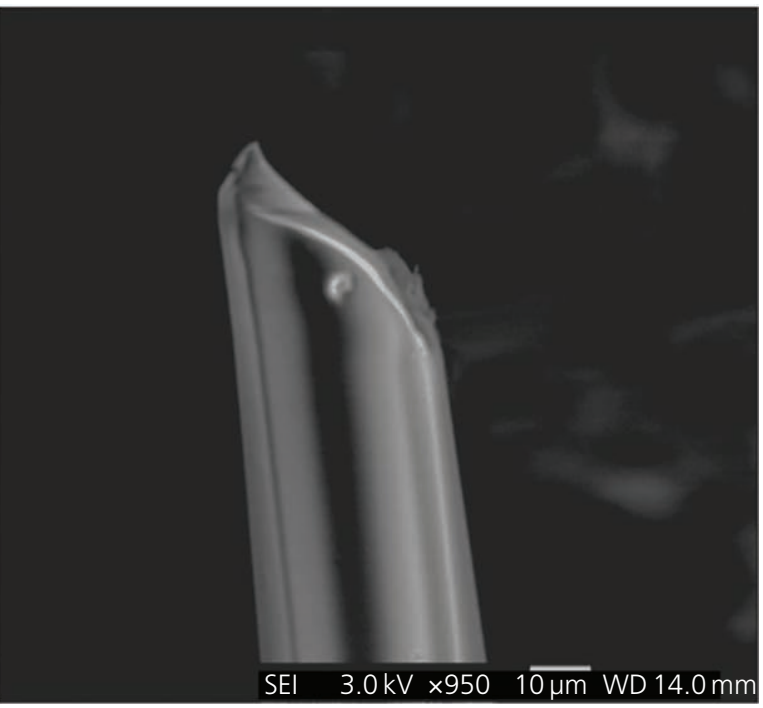

(d)

Figure 2. Oxidation and reduction properties of adrenaline using the FSCV protocol. (a) Two-dimensional hotspot, wherein current is represented on the $Y$-axis, while voltage is represented on the $X$-axis. The time axis is converted into a number of repetitions on the $Z$-axis. The hotspot shows the oxidation and reduction spots along with the steadiness of current across a number of trials. (b) Match between experimental and simulation data, showing that the experimental peaks are far broader. The experimental parameters were -0.4 to $1.3 \mathrm{~V}$ and cycled back to $-0.4 \mathrm{~V}$ at $600 \mathrm{~V} / \mathrm{s}$ repeated at $10 \mathrm{~Hz}$. The simulated data parameters were diffusion constant $D$ of $6 \times 10^{-6} \mathrm{~cm}^{2} / \mathrm{s}$, concentration of $1 \mu \mathrm{M}$ and number of electrons transported $(n)=2$; the rate of electron transport was set to $10^{-6} \mathrm{~cm}^{2} / \mathrm{s}$, and the chemical rate was $10^{-4} \mathrm{~cm}^{2} / \mathrm{s}$. (c, d) Examples of what the tips of the electrodes look like, showing how the end lies at an angle to the main axis

of peroxide, the authors varied the scan rate and measured the peak current. The peak current was proportional to the scan rate, showing

Table 1. Specific sensitivities of electrodes recently proposed in the literature for adrenaline sensing (approximate)

\begin{tabular}{lc} 
Reference & Sensitivity: $(\mu \mathbf{A} / \mathbf{m M}) / \mathbf{m m}^{2}$ \\
\hline Zare et al. ${ }^{31}$ & 0.2 \\
Reddy et al..$^{27}$ & 42 \\
Biswas et al. & 15.1 \\
Charitha and Manjunatha $^{28}$ & 275 \\
This work & 200
\end{tabular}

that peroxide is bound to the surface of the electrode (Figure 3(e)). The narrow oxidation peak at $0.85 \mathrm{~V}$ shows that oxidation of hydrogen peroxide is rapid and probably simple. ${ }^{5,6}$ Modelling the reaction shows the oxidation of hydrogen peroxide at $1.3 \mathrm{~V}$, using the parameters from the study by Spanos et al. ${ }^{5}$ (Figure 3(b)). However, upon increasing the rate of electron transfer, the authors are able to match the theory to experimental data (Figure 3(c)). This agrees with previous work using oxidised $\mathrm{CNTs}^{27,28,31}$ and also doped diamond ${ }^{30}$ that electron-transfer rates from monoamines are higher at doped carbon than at pure carbon. Those studies used electrochemical impedance spectroscopy using a ferri/ferrocyanide couple to remove the effect of the overpotential. 


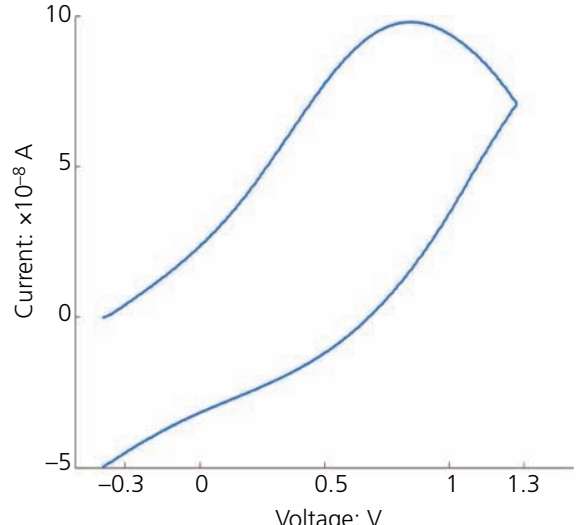

(a)

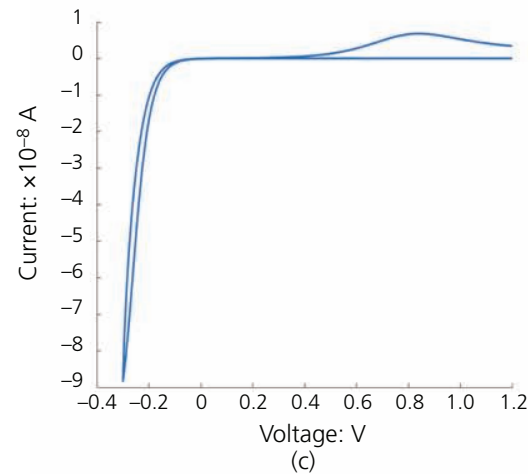

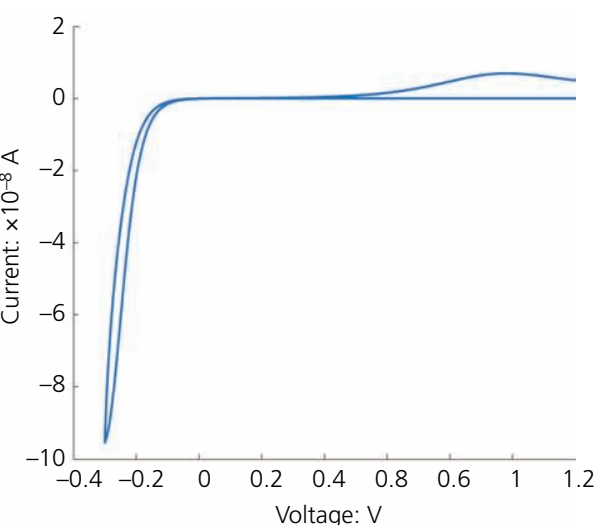

(b)

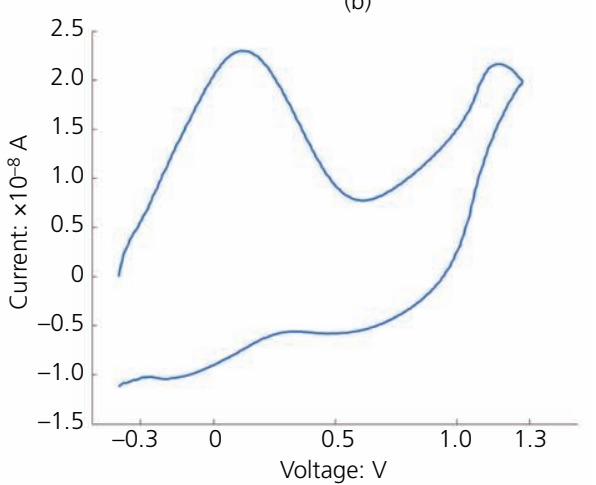

(d)

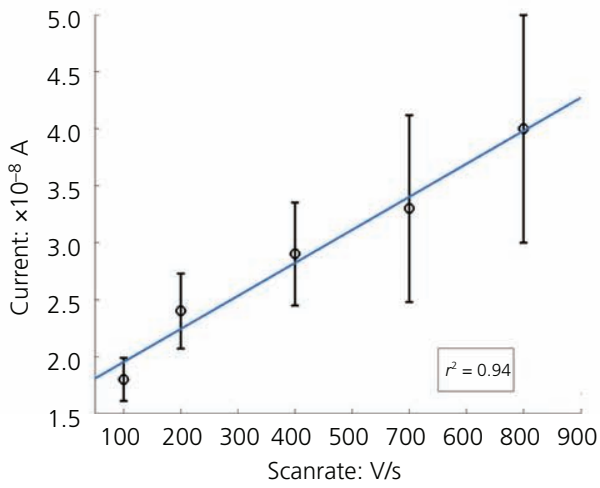

(e)

Figure 3. Cyclic voltammogram of $200 \mu \mathrm{M}$ hydrogen peroxide on a CNT-coated electrode. (a) Hydrogen peroxide oxidises at $0.85 \mathrm{~V}$. (b) Electrochemical modelling of the reaction. The diffusion coefficient was $2.5 \times 10^{-5} \mathrm{~cm}^{2} / \mathrm{s}$, the charge-transfer coefficient was 0.5 , the number of electron transfer $(n)=2$ and the electrochemical rate was set to $10^{-4} \mathrm{~cm} / \mathrm{s}$. (c) Increasing the rate of reaction to $10^{-6} \mathrm{~cm} / \mathrm{s}$ matches the experimental observation, thus suggesting that CNTs have a faster electron-transfer process. (d) Plot for $1 \mu \mathrm{M}$ adenosine, which shows an oxidation peak at $0.1 \mathrm{~V}$ and a second oxidation at $1.1 \mathrm{~V}$. Broad reduction peaks are seen at 0.7 and $0.2 \mathrm{~V}$. (e) Plot of the scan rate against the peak current for hydrogen peroxide. The electrode has a sensitivity of $11 \mathrm{nA} / \mu \mathrm{M}$

Adenosine interferes with the detection of hydrogen peroxide when measurements are conducted using normal carbon electrodes. To investigate the effect of adenosine, $1 \mu \mathrm{M}$ of adenosine was introduced in the flow cell chamber. Adenosine shows a broad oxidation peak at $0.2 \mathrm{~V}$ and another at $1.2 \mathrm{~V}$. Multiple reduction peaks can be observed at 0.6 and $0.2 \mathrm{~V}^{21}$ (Figure 3(d)). This suggests that pyrolytic carbon electrodes coated with CNTs have suitable overpotentials to separate the redox signals from analytes that have similar redox potentials on other substrates. Herein, the authors show that analytes with identical oxidation windows can be distinguished using CNT coatings onto pyrolytic electrodes. The authors' future work will be setting up a calibration method for multimodal analysis of analytes for detecting the detection limit of their sensor. 
The authors also intend to work on the reproducibility of the processes, which currently require each electrode to be calibrated individually due to variations in tip diameter and possibly angle. One possibility is to wet or dry etch the tips of the capillaries before and possibly also after the pyrolysis process.

\section{Conclusions}

In this work, it was shown that oxidised CNT-coated pyrolytic carbon electrodes can be used for the detection of adrenaline, hydrogen peroxide and adenosine and give different signals for them. The ability of pyrolytic-oxidised CNT electrodes to distinguish between analytes by shifting the potential at which they are oxidised coupled with the low overpotential for monoamines (reducing potential oxidative damage from solvolysis) makes them potentially useful surfaces for the electrochemical detection of neuromodulators. As mentioned earlier, the use of modified carbon electrodes, particularly for this type of measurement, is established, but most have concentrated on relatively large electrodes, ${ }^{30,31}$ paste electrodes ${ }^{28,29}$ and some contain metal particles. ${ }^{29}$

The authors' future work will include determining the oxidation potential of adrenaline on different surfaces using the FSCV method and investigating ways to deconvolute mixed signals, as despite the voltage shift of the analytes, they are all adsorbed on the electrode and give broad signals, so they are not fully resolvable yet. The interaction between the substrate in solution and the electrode seems to be very complex, and the authors feel that understanding it more would enable the electrode surface to be modified to maximise the number of sites where adrenaline can interact or to generate greater electrochemical differences between similar substrates such as adrenaline and serotonin. It would also apply to similar systems, such as that of polyphenols.

\section{REFERENCES}

1. Cechova S and Venton BJ (2008) Transient adenosine efflux in the rat caudate-putamen. Journal of Neurochemistry 105(4): 1253-1263.

2. Jacobs CB, Ivanov IN, Nguyen MD, Zestos AG and Venton BJ (2014) High temporal resolution measurements of dopamine with carbon nanotube yarn microelectrodes. Analytical Chemistry 86(12): 5721-5727.

3. Hashemi P, Dankoski EC, Petrovic J, Keithley RB and Wightman RM (2009) Voltammetric detection of 5-hydroxytryptamine release in the rat brain. Analytical Chemistry 81(22): 9462-9471.

4. Swamy BEK and Venton BJ (2007) Carbon nanotube-modified microelectrodes for simultaneous detection of dopamine and serotonin in vivo. Analyst 132(9): 876-884.

5. Spanos M, Gras-Najjar J, Letchworth JM et al. (2013) Quantitation of hydrogen peroxide fluctuations and their modulation of dopamine dynamics in the rat dorsal striatum using fast-scan cyclic voltammetry. ACS Chemical Neuroscience 4(5): 782-789.

6. Meunier CJ, Mitchell EC, Roberts JG et al. (2018) Electrochemical selectivity achieved using a double voltammetric waveform and partial least squares regression: differentiating endogenous hydrogen peroxide fluctuations from shifts in $\mathrm{pH}$. Analytical Chemistry 90(3): 1767-1776.

7. lijima S (2002) Carbon nanotubes: past, present, and future. Physica B: Condensed Matter 323(1-4): 1-5.

8. Ross AE and Venton BJ (2012) Nafion-CNT coated carbon-fiber microelectrodes for enhanced detection of adenosine. Analyst 137(13): 3045-3051.
9. Yang C, Trikantzopoulos E, Jacobs CB and Venton BJ (2017) Evaluation of carbon nanotube fiber microelectrodes for neurotransmitter detection: correlation of electrochemical performance and surface properties. Analytica Chimica Acta 965: 1-8.

10. Yang C, Jacobs CB, Nguyen MD et al. (2016) Carbon nanotubes grown on metal microelectrodes for the detection of dopamine. Analytical Chemistry 88(1): 645-652.

11. Yang C, Trikantzopoulos E, Nguyen MD et al. (2016) Laser treated carbon nanotube yarn microelectrodes for rapid and sensitive detection of dopamine in vivo. ACS Sensors 1(5): 508-515.

12. Schmidt AC, Wang $X$, Zhu $Y$ and Sombers LA (2013) Carbon nanotube yarn electrodes for enhanced detection of neurotransmitter dynamics in live brain tissue. ACS Nano 7(9): 7864-7873.

13. Kidokoro $Y$ and Ritchie AK (1980) Chromaffin cell action potentials and their possible role in adrenaline secretion from rat adrenal medulla. Journal of Physiology 307(1): 199-216.

14. Ambade V, Arora MM, Singh P, Somani BL and Basannar D (2009) Adrenaline, noradrenaline and dopamine level estimation in depression: does it help? Medical Journal Armed Forces India 65(3): 216-220.

15. Lee CR, Patel JC, O'Neill B and Rice ME (2015) Inhibitory and excitatory neuromodulation by hydrogen peroxide: translating energetics to information. Journal of Physiology 593(16): 3431-3446.

16. Rice ME (2011) $\mathrm{H}_{2} \mathrm{O}_{2}$ : a dynamic neuromodulator. Neuroscientist 17(4): 389-406.

17. Dringen R, Pawlowski PG and Hirrlinger J (2005) Peroxide detoxification by brain cells. Journal of Neuroscience Research 79(1-2): 157-165.

18. Petrovic J, Walsh PL, Thornley KT, Miller CE and Wightman RM (2010) Real-time monitoring of chemical transmission in slices of the murine adrenal gland. Endocrinology 151(4): 1773-1783.

19. Lee ST and Venton BJ (2018) Regional variations of spontaneous, transient adenosine release in brain slices. ACS Chemical Neuroscience 9(3): 505-513.

20. Ross AE and Venton BJ (2014) Sawhorse waveform voltammetry for selective detection of adenosine, ATP, and hydrogen peroxide. Analytical Chemistry 86(15): 7486-7493.

21. Heien MLAV, Phillips PEM, Stuber GD, Seipel AT and Wightman RM (2003) Overoxidation of carbon-fiber microelectrodes enhances dopamine adsorption and increases sensitivity. Analyst 128(12): 1413-1419.

22. Weese ME, Krevh RA, Li Y, Alvarez NT and Ross AE (2019) Defect sites modulate fouling resistance on carbon-nanotube fiber electrodes. ACS Sensors 4(4): 1001-1007.

23. Trautner E and Bradley T (1951) The early stages of the oxidation of adrenaline in dilute solution. Australian Journal of Scientific Research Series B 4(3): 303-343.

24. Chirila T, Cooper R and Constable I (1991) 'Black Prosthesis' revisited: a study of epinephrine-induced pigment deposits on poly (methyl methacrylate). Graefe's Archive for Clinical and Experimental Ophthalmology 229(6): 578-582.

25. Swamy BEK and Venton BJ (2007) Subsecond detection of physiological adenosine concentrations using fast-scan cyclic voltammetry. Analytical Chemistry 79(2): 744-750.

26. Nasirizadeh N, Shekari Z, Zare H et al. (2013) Electrosynthesis of an imidazole derivative and its application as a bifunctional electrocatalyst for simultaneous determination of ascorbic acid, adrenaline, acetaminophen, and tryptophan at a multi-wall carbon nanotubes modified electrode surface. Biosensors and Bioelectronics 41: 608-614.

27. Reddy KK, Satyanarayana M, Goud KY, Gobi KV and Kim H (2017) Carbon nanotube ensembled hybrid nanocomposite electrode for direct electrochemical detection of epinephrine in pharmaceutical tablets and urine. Materials Science and Engineering: C 79: 93-99.

28. Charithra MM and Manjunatha JG (2021) Electrochemical sensing of adrenaline using surface modified carbon nanotube paste electrode. Materials Chemistry and Physics 262: article 124293. 
29. Biswas S, Naskar H, Pradhan S et al. (2017) Simultaneous voltammetric determination of adrenaline and tyrosine in real samples by neodymium oxide nanoparticles grafted graphene. Talanta 206: article 120176.

30. Li YR, Li HJ, Li MJ and et al. (2017) Porous boron-doped diamond electrode for detection of dopamine and pyridoxine in human serum. Electrochimica Acta 258: 744-753.

31. Zare HR, Moradiyan B, Shekari Z and Benvidi A (2016) Application of 1-DOPA modified carbon nanotubes as a bifunctional electrocatalyst for simultaneous determination of ascorbic acid, adrenaline, acetaminophen and tyrosine. Measurement 90: 510-518.

32. Liu S, Ko A, Li W, Zhong W and Xing M (2014) NIR initiated and $\mathrm{pH}$ sensitive single-wall carbon nanotubes for doxorubicin intracellular delivery. Journal of Materials Chemistry B 2(9): 1125-1135.

33. Kole K (2015) Experience dependent plasticity of neurovascularisation. Journal of Neurophysiology 114(4): 2077-2079.

34. da Silva Lantyer A, Calcini N, Bijlsma A et al. (2018) A databank for intracellular electrophysiological mapping of the adult somatosensory cortex. Gigascience 7(12): article giy147.

35. Kole K, Zhang Y, Jansen EJR et al. (2020) Assessing the utility of Magneto to control neuronal excitability in the somatosensory cortex. Nature Neuroscience 23(9): 1044-1046.

36. Kole K and Celikel T (2019) Neocortical microdissection at columnar and laminar resolution for molecular interrogation. Current Protocols in Neuroscience 86(1): article e55.

37. Li S, Wang $H$, Young $M$ et al. (2018) Properties of electropolymerized dopamine and its analogues. Langmuir 35(5): 1119-1125.

38. Kund J, Daboss S, D'Alvise T et al. (2021) Physicochemical and electrochemical characterization of electropolymerized polydopamine films: influence of the deposition process. Nanomaterials 11(8): article 1964.
39. Thirumalraj B, Palanisamy S, Chen S and Kannan R (2016) Alumina polished glassy carbon electrode as a simple electrode for lower potential electrochemical detection of dopamine in its sub-micromolar level. Electroanalysis 28(3): 425-430.

40. Kole K, Scheenen W, Tiesinga P and Celikel T (2018) Cellular diversity of the somatosensory cortical map plasticity. Neuroscience \& Biobehavioral Reviews 84: 100-115.

41. Atcherley CW, Laude ND, Parent KL and Heien ML (2013) Fast-scan controlled-adsorption voltammetry for the quantification of absolute concentrations and adsorption dynamics. Langmuir 29(48): 14885-14892.

42. Yuan Z (2014) The effects of abrasive particles on the electrochemical behavior of adrenaline at different electrodes. Russian Journal of Electrochemistry 50(1): 80-86.

43. Yi Y, Weinberg G, Prenzel M et al. (2017) Electrochemical corrosion of a glassy carbon electrode. Catalysis Today 295: 32-40.

44. Venton BJ, Troyer KP and Wightman RM (2002) Response times of carbon fiber microelectrodes to dynamic changes in catecholamine concentration. Analytical Chemistry 74(3): 539-546.

45. Lin C, Chen L, Tanner EEL and Compton RG (2017) Electroanalytical study of dopamine oxidation on carbon electrodes: from the macro- to the micro-scale. Physical Chemistry Chemical Physics 20(1): 148-157.

46. Bacil RP, Chen L, Serrano SHP and Compton RG (2020) Dopamine oxidation at gold electrodes: mechanism and kinetics near neutral $\mathrm{pH}$. Physical Chemistry Chemical Physics 22(2): 607-614.

47. Choi J, Tojo S, Ahn DS et al. (2019) Proton transfer accompanied by the oxidation of adenosine. Chemistry - A European Journal 25(32): 7711-7718.

48. Schales O (1938) Empfindliche Methoden zum Nachweis von Wasserstoffperoxyd und seiner Entstehung bei einigen DehydrierungsVorgängen. Berichte der Deutschen Chemischen Gesellschaft 71(2): 447-460 (in German)

\section{How can you contribute?}

To discuss this paper, please submit up to 500 words to the journal office at journals@ice.org.uk. Your contribution will be forwarded to the author(s) for a reply and, if considered appropriate by the editor-in-chief, it will be published as a discussion in a future issue of the journal.

ICE Science journals rely entirely on contributions from the field of materials science and engineering. Information about how to submit your paper online is available at www.icevirtuallibrary.com/page/authors, where you will also find detailed author guidelines 